\title{
Was wären wir ohne Teamwork?
}

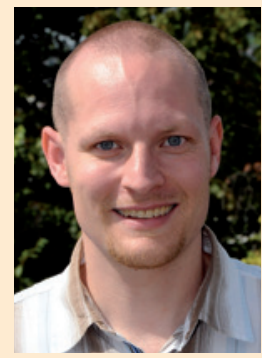

Christian Schäfer, Stuttgart
Die Frage lässt sich einfach beantworten: Uns - die Menschen - gäbe es nicht. Ohne einen engen Zusammenhalt wären die relativ kleinen Ursprungspopulationen unserer evolutionsgeschichtlich etwas näheren Vorfahren (Menschenaffenähnliche, Vormenschen, Urmenschen und anatomisch moderne Menschen) zu einem beliebigen Zeitpunkt sehr schnell von der Bildfläche verschwunden. Teamwork, soziale Interaktionen und all das, was daraus entstehen kann, machen auch die Basis unserer Kultur aus, mit der (Ur-)Menschen den Erdball erobert haben.

Auch unsere Sprache, Empathiefähigkeit und das Gespür für Musik haben das gegenwärtige Niveau erreicht, weil sie uns in der Vergangenheit einen erheblichen Vorteil im Zusammenleben und damit im Überleben sicherten. Und wer könnte sich menschliche Kulturen ohne diese Eigenschaften vorstellen? Eben! Auch die exzessive Entwicklung von immer komplexeren technischen Neuerungen sowie deren Weitergabe (Tradition) und Optimierung (kulturelle Evolution) gehören zu den Grundlagen für unser heutiges Verständnis des Menschseins. Diese waren und sind in der Gruppe mit u.a. der Möglichkeit zur Arbeitsteilung, zum Ideenaustausch und zum Füreinander-da-Sein im Verletzungs- und Krankheitsfall viel leichter zu realisieren als bei isolierten Individuen.

Das führt uns sofort zur Pflege und Medizin in der heutigen Zeit: Gerade bei der Betreuung von Nierenkranken sind viele Menschen beteiligt, nicht nur aus dem Pflegesektor und der Medizin, sondern z.B. auch aus der Ökotrophologie. Bei so vielen Akteuren inkl. des Patienten ist eine detaillierte Abstimmung und Aufgabenverteilung unumgänglich - eben Teamarbeit. Hierbei sollten genügend qualifizierte Mitarbeiter nicht aus Sparzwängen gegen weniger qualifizierte ausgetauscht werden, wie die Bundesarbeitsgemeinschaft Nephrologische Pflege (BANP) in ihrem Positionspapier ab Seite 242 in dieser Ausgabe der Dialyse aktuell u.a. klarstellt. Ansonsten ist das Patientenwohl gefährdet. Diese klare Positionierung entspringt einem Teamwork der Arbeitsgemeinschaft für nephrologisches Personal e.V. (AfnP) und dem Fachverband nephrologischer Berufsgruppen e. V. (fnb) - den beiden Verbänden hinter der BANP.

Teamarbeit ist auch in der Planung und Realisierung der verschiedenen Ausgaben der Dialyse aktuell notwendig. Ich bin sehr glücklich darüber, dass die Dialyse aktuell und die AfnP eine Organschaft verbindet. Dieser Zusammenarbeit entspringen so positive Dinge wie die Medienpartnerschaft des Thieme Verlags bei der Nephro Fachtagung Ulm und meine dortige Funktion als Programmbeirat sowie das Thieme Sponsoring des jährlich vergebenen Förderpreis Nephrologische Pflege (siehe S. 238) und meine dortige Funktion als Mitglied in der Jury, ganz zu schweigen von der regelmäßigen und gelungenen Kooperation bei der Gestaltung der Fachgesellschaftsseiten der AfnP. Ebenfalls sehr wichtig ist die Zusammenarbeit der Redaktion mit dem fnb, der BANP, dem Arbeitskreis Transplantationspflege e. V. (AKTX-Pflege) und der Deutschen Gesellschaft für Nephrologie e.V. (DGfN), welche die Dialyse aktuell als kooperierende Verbände begleiten. So können Sie regelmäßig interessante Neuigkeiten rund um diese Verbände lesen.

Besonders herausheben möchte ich die Trennung von Anzeigenakquise und Redaktion bei der Dialyse aktuell, die zusammen mit einem 2-fachen Review der Schwerpunktbeiträge die Unabhängigkeit sowie Qualität und somit auch die Vergabe von CME-Punkten garantiert. Pflegekräfte erhalten über das Abonnement oder den Bezug der Dialyse aktuell über eine Mitgliedschaft in der AfnP oder dem fnb jährlich 3 Punkte für die „Registrierung beruflich Pflegender“ - ein weiteres Qualitätsmerkmal der Zeitschrift.

Der aktuelle Schwerpunkt „Begleittherapien bei Nierenkranken“ ist das Ergebnis einer sehr guten Teamarbeit aller Beteiligten. Auch bei Therapien kann man das Konzept der Zusammenarbeit anwenden: Ohne begleitende Behandlungen (also rein „klassisch“ behandelt) geht es den Patienten schlechter, man muss den Nierenkranken also möglichst ganzheitlich betrachten. Was bleibt? Vielleicht dies: Teamwork ist eine zutiefst menschliche Eigenschaft und sollte daher auch und gerade in der Nephrologie Anwendung finden. Ich wünsche Ihnen eine angenehme Lektüre dieser Ausgabe der Dialyse aktuell! 